\title{
An algorithm for pulsed activation of solenoid valves for variable rate application of agricultural chemicals
}

\begin{abstract}
An alternative algorithm to Pulse Width Modulation (PWM) for pulsed activation of solenoid valves for applying chemicals through agricultural sprayer nozzles is presented. Solenoid valves attached to individual spray nozzles on a modified EMDEK tractor-mounted sprayer system are activated by electronic pulsation to vary the application rate of agricultural chemicals, varying the output by location according to Geographical Information System (GIS) data and a GPS system. A potential advantage of this alternative algorithm over pulse width modulation based systems is the use of lower-cost industrial solenoid valves with slower opening and closing times instead of the more expensive high speed valves normally used in PWM systems.
\end{abstract}

